ARTICLE

\title{
Common variants in ZMIZ1 and near NGF confer risk for primary dysmenorrhoea
}

\author{
Zhiqiang $\mathrm{Li}^{1,2,3,4, \star}$, Jianhua Chen ${ }^{2,3,5, \star}$, Ying Zhao ${ }^{2,6, \star}$, Yujiong Wang ${ }^{7}$, Jinrui $\mathrm{Xu}^{7}$, Jue $\mathrm{Ji}^{2}$, Jingyi Shen ${ }^{2}$, \\ Weiping Zhang ${ }^{8}$, Zuosong Chen ${ }^{6}$, Qilin Sun ${ }^{6}$, Lijuan Mao ${ }^{9}$, Shulin Cheng ${ }^{6}$, Bo Yang7 ${ }^{7}$ Dongtao Zhang ${ }^{7}$, \\ Yufeng $\mathrm{Xu}^{2}$, Yingying Zhao ${ }^{2}$, Danping Liu ${ }^{2}$, Yinhuan Shen ${ }^{2}$, Weijie Zhang ${ }^{2}$, Changgui Li ${ }^{10}$, Jiawei Shen ${ }^{2}$ \\ \& Yongyong Shi ${ }^{1,2,3,4,5,11}$
}

Primary dysmenorrhoea, defined as painful menstrual cramps in the absence of pelvic pathology, is a common problem in women of reproductive age. Its aetiology and pathophysiology remain largely unknown. Here we performed a two-stage genome-wide association study and subsequent replication study to identify genetic factors associated with primary dysmenorrhoea in a total of 6,770 Chinese individuals. Our analysis provided evidence of a significant $\left(P<5 \times 10^{-8}\right)$ association at rs76518691 in the gene ZMIZ1 and at rs7523831 near NGF. ZMIZ1 has previously been associated with several autoimmune diseases, and NGF plays a key role in the generation of pain and hyperalgesia and has been associated with migraine. These findings provide future directions for research on susceptibility mechanisms for primary dysmenorrhoea. Furthermore, our genetic architecture analysis provides molecular support for the heritability and polygenic nature of this condition.

\footnotetext{
${ }^{1}$ The Affiliated Hospital of Qingdao University \& The Biomedical Sciences Institute of Qingdao University (Qingdao Branch of SJTU Bio-X Institutes), Qingdao University, Qingdao 266003, China. ${ }^{2}$ Bio-X Institutes, Key Laboratory for the Genetics of Developmental and Neuropsychiatric Disorders (Ministry of Education), the Collaborative Innovation Center for Brain Science, Shanghai Jiao Tong University, Shanghai 200030, China. ${ }^{3}$ Institute of Social Cognitive and Behavioral Sciences, Shanghai Jiao Tong University, Shanghai 200240, China. ${ }^{4}$ Institute of Neuropsychiatric Science and Systems Biological Medicine, Shanghai Jiao Tong University, Shanghai 200042, China. ${ }^{5}$ Shanghai Key Laboratory of Psychotic Disorders, Shanghai Mental Health Center, Shanghai Jiao Tong University School of Medicine, Shanghai 200030, China. ${ }^{6}$ Physical Education Department, Shanghai Jiao Tong University, Shanghai 200042, China.

${ }^{7}$ Key Laboratory of Ministry of Education for Conservation and Utilization of Special Biological Resources in the Western China, Ningxia University, Yinchuan 750021, China. ${ }^{8}$ Campus Health Center of Shanghai Jiao Tong University, Shanghai 200042, China. ${ }^{9}$ School of Physical Education and Coaching, Shanghai University of Sport, Shanghai 200438, China. ${ }^{10}$ Shandong Provincial Key Laboratory of Metabolic Disease \& the Metabolic Disease Institute of Qingdao University, Qingdao 266003, China. ${ }^{11}$ Changning Mental Health Center, Shanghai 200042, China. * These authors contributed equally to this work. Correspondence and requests for materials should be addressed to Y.S. (email: shiyongyong@gmail.com).
} 
P rimary dysmenorrhoea is a medical condition characterized by menstrual pain without any evident pelvic pathology ${ }^{1}$. It causes significant disruption in quality of life and can result in absenteeism. It is thought to be the most common gynaecologic complaint among women, with a prevalence estimated at nearly $50 \%$ in menstruating females ${ }^{2}$. The causes of primary dysmenorrhoea are not precisely understood. Both emotional or psychological and physiological factors have been considered causes of primary dysmenorrhoea ${ }^{3}$, with anxiety ${ }^{4}$, stress $^{5}$ and prostaglandin production ${ }^{6,7}$ reported as contributing factors. Twin studies suggest that primary dysmenorrhoea is heritable $e^{8-10}$. However, very few specific genetic variants have been found to be associated with primary dysmenorrhoea ${ }^{11,12}$, and these studies had small sample sizes. Thus, the genetic cause and mechanism of primary dysmenorrhoea are largely unknown. The discovery of genetic variants associated with primary dysmenorrhoea may lead to insights regarding its underlying biological mechanisms and could facilitate management of the condition (including the selection of medical and relief treatments).

Here we performed a two-stage genome-wide association study and subsequent replication study of primary dysmenorrhoea using 6,770 Chinese individuals. We identified two susceptibility loci in ZMIZ1 and near NGF for primary dysmenorrhoea, and provided further genetic evidence for the polygenic nature of primary dysmenorrhoea. Our study provides new insights into the genetic architecture of primary dysmenorrhoea and suggests directions for future research.

\section{Results}

Primary dysmenorrhoea GWAS susceptibility loci. In the discovery phase, a genome-wide association study (GWAS) meta-analysis was performed across three cohorts comprising a total of 5,324 individuals (2,404 cases and 2,920 controls; Supplementary Tables 1 and 2). Principal component analysis (PCA) was applied to identify population substructures within the samples (Supplementary Fig. 1). To enable integrative and cross-platform analysis, genotype imputations were employed in all studies using the reference data from the 1000 Genomes Project March 2012 release. Association analyses with adjustments for potential population stratification were performed at the cohort level. The results were combined using inversevariance meta-analysis based on the fixed-effect model. A total of $6,277,361$ single-nucleotide polymorphisms (SNPs) passed quality control and were kept for further analysis. A genomic inflation factor of 1.018 was observed, suggesting that the confounding effects of population stratification were well controlled (Supplementary Fig. 2). From the discovery stage, no genomewide significant (GWS) locus $\left(P<5 \times 10^{-8}\right)$ was identified (Supplementary Fig. 3). We then took the SNP with the lowest $P$ value among the 16 loci with $P<1 \times 10^{-5}$ for replication in an independent cohort of 1,446 individuals (678 cases and 768 controls, Supplementary Tables 1 and 3). In a subsequent metaanalysis of the discovery and replication samples, we identified two GWS SNPs: rs76518691 $\left(P=1.47 \times 10^{-9}\right)$, an intron variant in $Z M I Z 1$, and $\operatorname{rs7523831}\left(P=1.36 \times 10^{-8}\right)$, located close to NGF (Table 1, Figs 1 and 2). For both of the GWS SNPs, there was no evidence to suggest significant heterogeneity across discovery and replications ( $P_{\text {het }}>0.05$, Supplementary Table 3 ).

Functional implications of the susceptibility loci. To explore the potential implications and epigenetic profile of the association signals, we queried annotations of the non-coding genome for the index SNPs and those in linkage disequilibrium (LD) with them $\left(r^{2}>0.8\right.$, based on the 1000 Genomes Project ASN data set).
Many of these SNPs, including the significant SNPs rs76518691 and rs7523831, are predicted to reside within promoter and/or enhancer elements, suggesting putative regulatory functionality for these loci (Supplementary Table 4). Further tissuespecific regulatory analysis using the Roadmap Epigenome and ENCODE integrated data sets highlighted several tissues and cell types, including adipose-derived mesenchymal stem cells (AD-MSC; H3K4me3 and H3K4me1 signals at the region surrounding rs76518691 and $\mathrm{H} 3 \mathrm{~K} 4 \mathrm{me} 3$ signal at the region surrounding rs7523831), bone marrow-derived mesenchymal stem cell (BD-MSC) culture cells (H3K4me3 and H3K27ac signals for rs7523831), cortex and ganglionic eminence-derived neurospheres (H3K4mel signal for rs7523831) and some musclerelated tissues (Supplementary Figs 4 to 9). Some of these cell types might be biologically relevant. For instance, human AD-MSCs have been shown to promote neurogenesis ${ }^{13}$ and to play a neuroprotective role $^{14}$ in animal studies. In addition, human BM-MSCs and AD-MSCs were found to downregulate inflammatory and $\mathrm{T}$-cell responses in vitro ${ }^{15,16}$. Both the cortex and ganglionic eminence are regions of the nervous system where pain is mediated through complicated processing pathways ${ }^{17}$.

To assist in interpreting and contextualizing the results, we investigated whether the loci associated with primary dysmenorrhoea were previously suspected of being involved in the aetiology of other diseases or traits and conducted functional annotation searches of published literature. We reasoned that any prior knowledge might help further unravel the potential roles of the loci. Variants in or near ZMIZ1 have been found to be associated with several complex diseases or traits (Supplementary Table 5), including multiple autoimmune diseases (that is, inflammatory bowel disease, multiple sclerosis, celiac disease, vitiligo, Crohn's disease and psoriasis). ZMIZ1 encodes the zinc finger protein MIZ type 1 , which regulates TGF-beta/SMAD signalling ${ }^{18}$ to play critical roles in the immune system ${ }^{19}$. A variant near NGF is associated with migraine (Supplementary Table 5), a major headache disorder. Most recently, a GWAS in female individuals of European descent identified NGF as pain-severity-associated locus in dysmenorrhoea (regardless of primary or secondary types $)^{20}$. NGF encodes a secreted protein that possesses nerve growth stimulating activity and is critical for the survival and maintenance of sympathetic and sensory neurons. In addition, NGF exerts biological effects on a variety of immune system cells ${ }^{21}$ and contributes to the immune response ${ }^{22}$ and is involved in a number of autoimmune disorders such as rheumatoid arthritis ${ }^{23}$ and psoriasis ${ }^{24}$. Moreover, prostaglandins, physiological factors probably involved in primary dysmenorrhoea, are thought to be powerful inducers of $\mathrm{NGF}^{25}$. Under inflammatory conditions, the NGF level increases and induces axonal outgrowth in nearby pain neurons, which leads to increased pain perception ${ }^{26}$.

Genome-wide gene and pathway analysis. To leverage our genome-wide data set and interpret the results, we conducted complementary gene-based and pathway-based association analyses. In the gene-based analysis, none of the tested genes reached significance after Bonferroni correction $\left(P<2 \times 10^{-6}\right)$, and the most associated gene was IL1A $\left(P=2.60 \times 10^{-5}\right.$, Supplementary Table 6). IL1A encodes a pleiotropic cytokine that is a member of the interleukin-1 cytokine family. This cytokine is involved in various inflammatory processes and hematopoiesis and plays one of the most important roles in the regulation of the immune response. We also identified five gene sets that were enriched at a false discovery rate $<0.05$, the top three of which included interleukin-1 receptor binding, the amylase family and the bone morphogenic protein (BMP) signalling pathway (Supplementary Table 7). 
Table 1 | Results for the two genome-wide significant SNPs.

\begin{tabular}{|c|c|c|c|c|}
\hline Info & Effect allele (Freq.) & Phase & OR $(95 \% \mathrm{CI})$ & $P$ value \\
\hline rs7523831 & $C(0.543)$ & Data set 1 & $0.753(0.662-0.857)$ & $1.66 \times 10^{-5}$ \\
\hline Chr1: 115,824,192 & & Data set 2 & $0.952(0.822-1.102)$ & 0.5074 \\
\hline NGF & & Data set 3 & $0.842(0.739-0.958)$ & $9.27 \times 10^{-3}$ \\
\hline \multirow[t]{3}{*}{ Downstream } & & Discovery & $0.837(0.775-0.905)$ & $6.94 \times 10^{-6}$ \\
\hline & & Data set 4 & $0.762(0.659-0.882)$ & $2.70 \times 10^{-4}$ \\
\hline & & Combined & $0.820(0.766-0.878)$ & $1.36 \times 10^{-8}$ \\
\hline rs76518691 & $A(0.133)$ & Data set 1 & $0.769(0.638-0.927)$ & $5.99 \times 10^{-3}$ \\
\hline Chr10: $80,918,767$ & & Data set 2 & $0.667(0.539-0.824)$ & $1.79 \times 10^{-4}$ \\
\hline ZMIZ1 & & Data set 3 & $0.770(0.629-0.943)$ & 0.0114 \\
\hline \multirow[t]{3}{*}{ Intron } & & Discovery & $0.738(0.657-0.828)$ & $2.31 \times 10^{-7}$ \\
\hline & & Data set 4 & $0.696(0.556-0.871)$ & $1.55 \times 10^{-3}$ \\
\hline & & Combined & $0.729(0.658-0.807)$ & $1.47 \times 10^{-9}$ \\
\hline
\end{tabular}

Freq., the frequency for the effect allele; OR, odd ratio; SNP, single-nucleotide polymorphism; $95 \% \mathrm{Cl}$, 95\% confidence interval. Info, information for SNP (SNP id, chromosome: position, gene and location). The chromosomal positions are based on hg19. Genome-wide significant $\left(P<5 \times 10^{-8}\right)$ results are shown in bold.

SNP heritability of primary dysmenorrhoea. To determine the proportion of variation in susceptibility to primary dysmenorrhoea that is captured by common variants, we used the restricted maximum likelihood method to estimate the total variance explained by the genotyped and imputed SNPs $\left(h_{g}^{2}\right)$ using the discovery cohort data sets. We observed an $h_{g}^{2}$ of $22.9 \%$ (s.e. $=5.8 \%$ ) from the autosomal SNPs. We also sought to partition the heritability across functional categories to assess enrichment. Of the six primary functional annotation categories proposed by Gusev et al. ${ }^{27}$, the highest enrichment was observed for coding variants, which explained $24.0 \%$ (s.e. $=17.8 \%$ ) of the $h_{g}^{2}$ within $1.5 \%$ of SNPs $(16.3 \times$ enrichment $)$. DNaseI hypersensitivity sites (DHSs) spanned $16.1 \%$ of SNPs but explained approximately $60.9 \%$ (s.e. $=46.7 \%)$ of the $h_{g}^{2}(3.8 \times$ enrichment), and no enrichment was observed for the other four categories (Supplementary Table 8).

Effects of endometriosis variants on primary dysmenorrhoea. Endometriosis is the most common cause of secondary dysmenorrhoea. Several GWASs have been conducted on patients with endometriosis, and six GWS loci have been identified (Supplementary Table 9). We investigated whether the previously identified endometriosis risk alleles also confer risk to primary dysmenorrhoea. Of the seven GWS SNPs, one (rs1537377) showed nominal significant association but in the opposite direction of our GWAS discovery samples (Supplementary Table 10). However, we also noted that a SNP (rs6542095) near IL1A (the most significantly associated gene from our gene-based analysis) was found to be associated near GWS with endometriosis $\left(P=3.45 \times 10^{-7}\right)$ and at GWS with moderate-to-severe endometriosis $\left(P=3.43 \times 10^{-8}\right)^{28}$. We observed association between rs6542095 and primary dysmenorrhoea with a $P$ value of $1.71 \times 10^{-4}$, and the risk allele showed directional consistency across diseases.

\section{Discussion}

Primary dysmenorrhoea, which affects $>50 \%$ of menstruating women, causes extensive personal and public health problems, yet knowledge of its pathophysiology and aetiology is limited. Genome-wide association analysis is a hypothesis-free approach that has been widely and successfully applied in genetic analysis of complex traits and diseases ${ }^{29}$. In this study, we adopted a two-stage design analysis: we followed the initial genome-wide genotyping of 2,404 cases and 2338 controls by testing the top most associated SNPs from the discovery stage in an independent replication cohort of 678 cases and 768 controls. We identified two GWS association signals at rs76518691 (in ZMIZ1) and rs7523831 (near NGF) in the joint analysis of the discovery and replication cohorts. We also conducted supplemental analyses to aid in interpreting the results. Functional annotation suggested potential regulatory functionality for both loci, and cell-type specific analysis highlighted chromatin mark (for example, H3K4me3, H3K4me1 and so on) signals at some possible biologically relevant tissues, including AD-MSCs, BM-MSCs and the cortex and ganglionic eminence. ZMIZ1 has previously been associated with multiple autoimmune diseases. NGF has been shown to play a critical role in the generation of pain and has been associated with migraine and pain severity in dysmenorrhoea. NGF is also known to be involved in some autoimmune disorders. Previous researches demonstrated obvious immunological functions for both ZMIZ1 and NGF, suggesting a potential role for the immune system in the pathogenesis of primary dysmenorrhoea.

The gene-based and pathway-based analyses also supported a role of the immune system in primary dysmenorrhoea. In the gene-based analysis, the top significant gene is IL1A, which encodes a key regulator of immune and inflammatory processes. The pathway analysis highlighted several gene sets, including interleukin-1 receptor binding and the BMP signalling pathway. Interleukin-1 receptor binding plays an important role in mediating many immune and inflammatory responses ${ }^{30}$, while the BMP signalling pathway is thought to have important roles in the regulation of cell survival, proliferation and differentiation in the immune system ${ }^{31}$. It should be mentioned that $I L 1 A$ has been robustly implicated in endometriosis ${ }^{28,32}$, which is the most common cause of secondary dysmenorrhoea. However, we did not observe any other clear evidence for shared genetic backgrounds between endometriosis and primary dysmenorrhoea in the previously reported GWAS findings. Thus, further studies are required to fully clarify the relationship between these two diseases.

We estimated that $\sim 23 \%$ of the variance in primary dysmenorrhoea can be explained by the autosomal SNPs. Of them, the DHS and coding variants explained about $61 \%$ and $24 \%$ of $h_{g}^{2}$, respectively. In the context of the GWAS literature, the estimated $h_{g}^{2}$ for primary dysmenorrhoea was comparable to the values observed for other complex traits and diseases, such as body mass index $(\sim 16 \%)^{33}$ and schizophrenia $(\sim 23 \%)^{34}$. For partitioned heritability for specific functional categories, enrichment was observed for the DHSs category (explaining over $50 \%$ of the estimated $h_{g}^{2}$ ), similar to other common diseases ${ }^{27}$. 

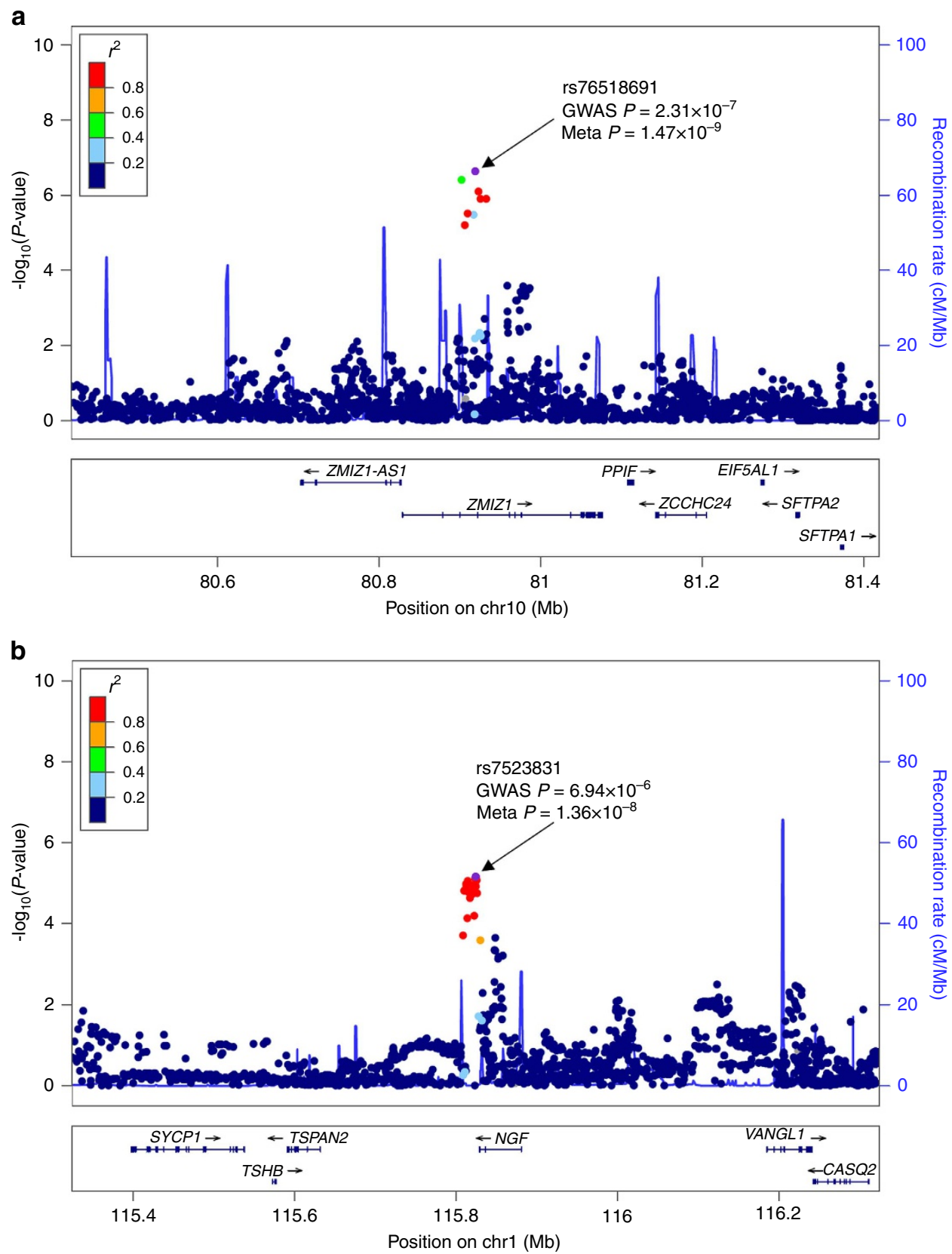

Figure 1 | Regional association plots of loci associated with primary dysmenorrhoea. (a) rs76518691 and (b) rs7523831. Purple circles represent the most significantly associated SNP (marker SNP) in each region in the meta-analysis of discovery and replication. $-\log 10 P$ values ( $y$ axis) of the SNPs (within the regions spanning $500 \mathrm{~kb}$ on either side of the marker SNP) are presented according to the chromosomal positions of the SNPs ( $x$ axis, hg19). SNPs are coloured according to their linkage disequilibrium (LD) with the marker SNP. The LD values were established based on the 1000 Genome Asian (ASI) data (March 2012). Estimated recombination rates with samples from the 1000 Genomes Project March 2012 release are shown as blue lines, and the genomic locations of genes within the interested regions annotated from the UCSC Genome Browser are shown as arrows.

Our findings suggest that primary dysmenorrhoea is a highly polygenic condition.

There are some limitations in this study. In our two-stage GWAS with multiple data sets, the two highlighted SNPs reached GWS only after joint analysis of the discovery and replication cohorts, especially for rs7523831 with 'Data set 2' producing a non-significant $P$ value $(>0.05)$. Nonetheless, the association signals for both GWS SNPs are consistent across at least four data sets, so that they are unlikely to be false positives. Therefore, we believe that the sample size in this study is still relatively small and the statistical power is limited. Moreover, on the clinical settings, the secondary dysmenorrhoea can only be ruled out by abdominal imaging examination. Thus, it is conceivable that the certain portion of the case group could be contaminated by the secondary aetiologies, which might impose some subtle influence on the final results.

In summary, we present the first GWAS for primary dysmenorrhoea in Chinese population and identify two GWS loci (ZMIZ1 and NGF). The results provide further genetic evidence for the polygenic nature of primary dysmenorrhoea and show that a remarkable proportion of heritability can be captured by common SNPs. Our findings shed light on the 
a

Data set

Odds ratio $(95 \% \mathrm{Cl})$

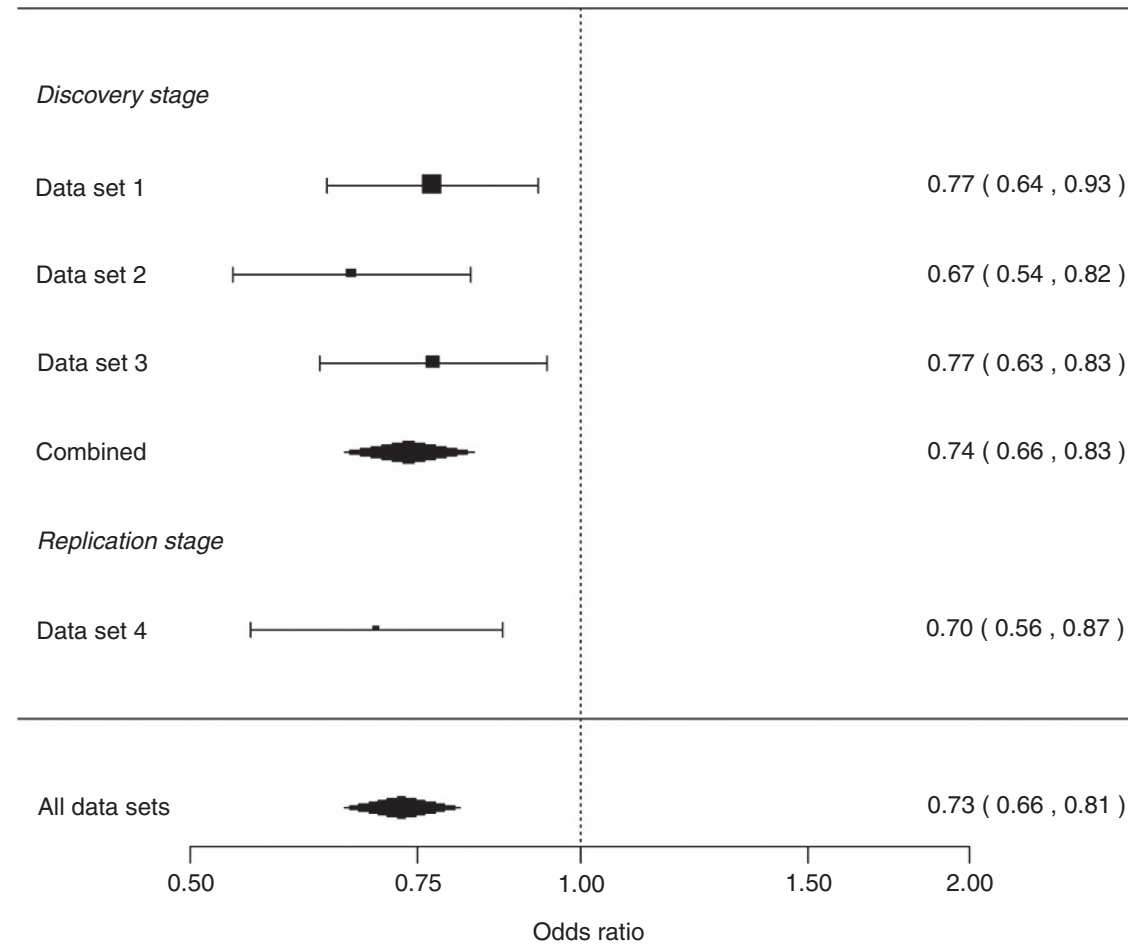

b

$\begin{array}{lll}\text { Data set } & \text { rs7523831 Odds ratio }(95 \% \mathrm{Cl})\end{array}$

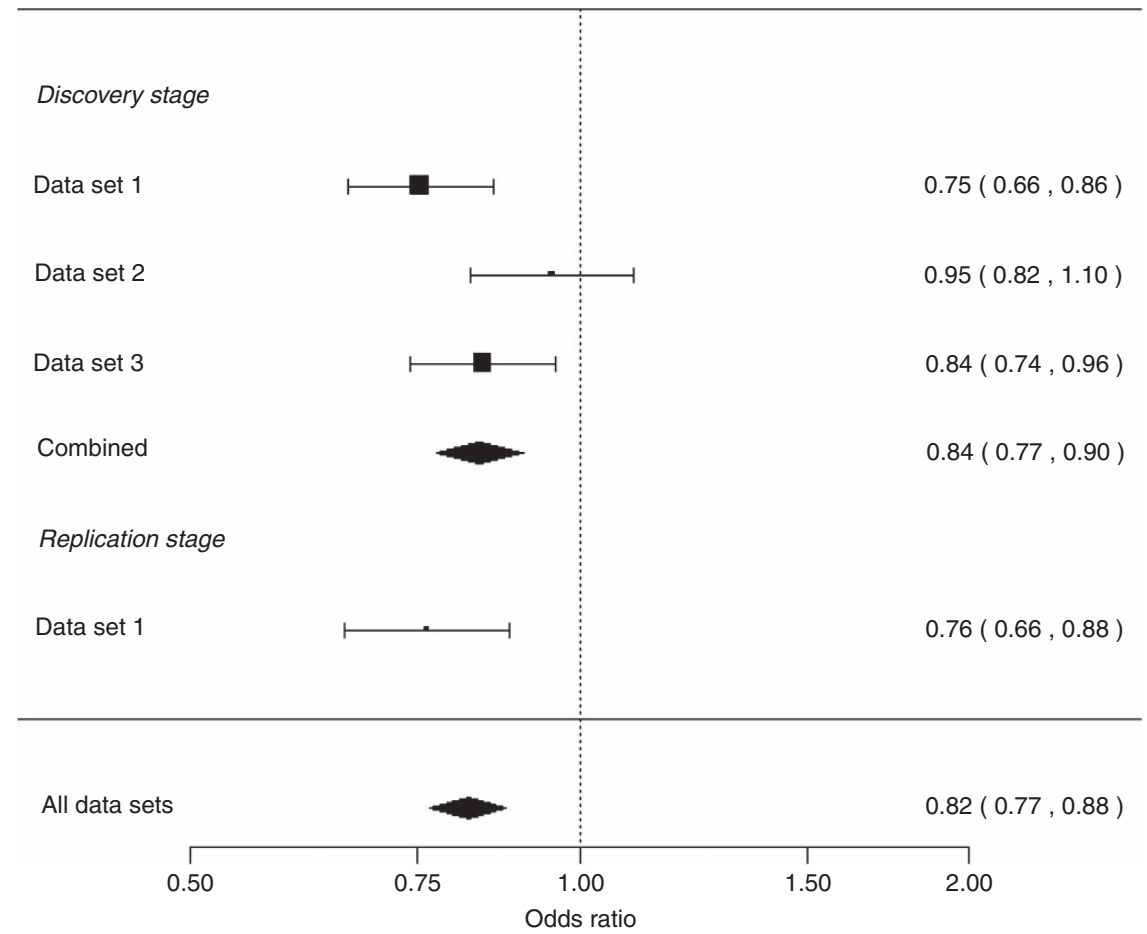

Figure 2 | Forest plots for the two genome-wide significant SNPs. rs76518691 (a) and rs7523831 (b). The plots show the subset-specific odds ratios and 95\% confidence intervals for all data sets in the discovery and replication stages presented as rectangles and bars, respectively. For each SNP, the association estimate and confidence interval for the fixed-effect meta-analysis combining discovery stage and all data sets results are presented as a diamond.

genetic architecture of primary dysmenorrhoea and suggest future directions for research on susceptibility mechanisms. Additional GWASs involving multiple ethnic populations and functional studies are necessary to identify additional genetic factors and unravel the complex biology of primary dysmenorrhoea. 


\section{Methods}

Study design. We performed a two-stage GWAS to identify loci associated with primary dysmenorrhoea in Chinese females. In the discovery stage, we performed a meta-analysis of three GWAS data sets (2,404 cases and 2,920 controls in total). The top significant variants were further tested in an independent data set (678 cases and 768 controls). All participants provided written informed consent. Approval was obtained from the Ethics Committee of Human Genetic Resources in Bio-X Institutes at Shanghai Jiao Tong University. We confirm that our study is compliant with the 'Guidance of the Ministry of Science and Technology for the Review and Approval of Human Genetic Resources'. The descriptive statistics of the samples are provided in Supplementary Table 1.

Phenotype. Dysmenorrhoea (or painful menstruation, MeSH ID: D004412) is a medical condition involving menstrual pain. Dysmenorrhoea is commonly divided into primary and secondary dysmenorrhoea on the basis of pathophysiology. Secondary dysmenorrhoea is associated with an identifiable disease (such as endometriosis), whereas primary dysmenorrhoea occurs without pelvic pathology ${ }^{1}$. In adolescents and young adults, the most prevalent type is primary dysmenorrhoea. A medical history and physical examination are usually sufficient to make a diagnosis of primary dysmenorrhoe ${ }^{3}$. We investigated $\sim 10,000$ female students at Shanghai Jiao Tong University and Ningxia University by routine physical examination during a period spanning 2008-2014, and most of the participants were born over a period of 17 years (from 1980 to 1996) and ranging from 18 to 34 years of age at the time of sampling. A visual analogue scale has previously been used to rate menstrual pain ${ }^{35,36}$. In this study, we adopted a horizontal $10-\mathrm{cm}$ visual analogue scale with endpoints spanning from 'no pain at all' (score $=0$ ) on the far left to 'the worst pain' (score $=10)$ on the far right. Scores of less than 1 were assigned to a control group, and scores higher than 4 (moderate intensity) ${ }^{36}$ were assigned to the case group. The participants with possible causes of secondary dysmenorrhoea, such as endometriosis and other gynaecological problems ${ }^{7}$, were excluded. A total of 6,770 individuals were genotyped and analysed in this GWAS.

Genotyping and quality control (QC). For data set 1 , genotyping was performed using Illumina HumanOmni1-Quad BeadChips according to the manufacturer's instructions (Illumina, Inc., San Diego, CA, USA). Genotype calls were generated using Genome Studio software. For data sets 2 and 3, genotyping was performed using the Affymetrix Axiom Genome-Wide CHB1 \& CHB2 Array Plate Set according to the manufacturer's protocols (Affymetrix, Inc., Santa Clara, CA, USA). Genotype calls for the CHB1 and CHB2 arrays were generated separately according to the Axiom Genotyping Solution Data Analysis Guide. For data set 4, genotyping was performed using the Sequenom MassARRAY iPLEX platform following the manufacturer's instructions (Sequenom, Inc., San Diego, CA, USA). Genotype calls were made using SEQUENOM's Typer 4.0 software.

Systematic QC analysis at both the sample and SNP levels was performed separately for each GWAS data set. We first filtered out low-quality samples: samples with a call rate $<97 \%$ in data set 1 , and samples with a dish QC value $<0.82$ in data sets 2 and 3 . Next, gender was estimated via genotyping data, and samples with inconsistent gender were removed. Heterozygosity rates were calculated for each sample, and deviations of more than 6 s.d. from the mean were excluded. PLINK's identity-by-descent (IBD) analysis was used to detect cryptic relatedness. The member (with a lower call rate) of the pair of unexpected duplicates or probable relatives (PI_HAT $>0.20$ ) was also excluded. For SNP QC, SNPs with call rates $<97 \%$, MAF $<3 \%$ or significant deviations from HardyWeinberg equilibrium (HWE) in the controls (HWE $P \leq 1 \times 10^{-6}$ ) were excluded. Subsequently, PCA was performed using a linkage disequilibrium (LD)-pruned SNP set to identify population substructures and outliers, which were removed. Detailed information regarding QC procedures is provided in Supplementary Table 2.

Imputation. Ungenotyped variants were imputed to the post-quality control GWAS data using SHAPEIT 2.0 (phasing step) ${ }^{37}$ and IMPUTE2 (imputation step) $^{38}$ software. The haplotype information was obtained from the 1000 Genomes Project (Phase I integrated variant set across all 1,092 individuals, v2, March 2012) ${ }^{39}$.

Population stratification analysis. Population stratification was assessed using a PCA-based method implemented in the software package EIGENSTRAT ${ }^{40}$, and a total of 20 principal components were generated.

Association and meta-analysis. SNPTEST ${ }^{41}$ was used for single SNP association using a case-control test assuming an additive genetic model. The first principal component from PCA analysis was included to control for unobserved population structure. For the replication study, allelic association analysis was conducted using SHEsis ${ }^{42,43}$. An inverse-variance method (fixed-effect model)-based meta-analysis was adopted to combine results from different data sets, and heterogeneity across the data sets was evaluated using Cochran's $Q$ test, which was implemented in META ${ }^{44}$.
Bioinformatic analysis and functional annotation of GWS loci. The genomewide significant SNPs and variants in LD $\left(r^{2}>0.8\right.$ in the Asian 1000 Genomes Project population) were annotated using HaploReg ${ }^{45}$ and RegulomeDB ${ }^{46}$. These functional annotations include promoter and enhancer histone marks, DHSs, bound proteins, and altered motifs from the ENCODE ${ }^{47}$ and Roadmap Epigenomics Projects ${ }^{48}$, as well as GERP and SiPhy conservation scores. The Roadmap Epigenome Browser was used to explore the tissue-specific regulatory roles of genome-wide significant $\mathrm{SNPs}^{49}$. The integrated data from both the ENCODE and Roadmap Epigenomics Consortium Projects for histone profiles (H3K4me1, H3K4me3 and H3K27ac) of 46 primary human tissues and cells were analysed.

We extracted previously reported GWAS associations within $500 \mathrm{~kb}$ of the top significant SNPs from the NHGRI GWAS Catalog ${ }^{50}$, and we searched the published literature in PubMed for related genes to obtain additional functional evidence for these SNPs and genes.

Gene- and pathway-based association analyses. We performed gene-based association testing using VEGAS2 (ref. 51) with the gene boundary option of '0kbloc' for SNP selection. We used MAGENTA ${ }^{52}$ to explore pathway-based associations in the GWAS meta-analysis data set (excluding the MHC region due to difficulties in accounting for the gene density and LD pattern $s^{53}$ ). An individual pathway that reached a false discovery rate $q<0.05$ in either analysis (95th and 75th percentiles cutoff) was considered to be suggestively enriched.

Analysis of heritability. The proportion of variance to primary dysmenorrhoea explained by the common SNPs was estimated using a tool for genome-wide complex trait analysis (GCTA) ${ }^{54}$. For functional partitioning of SNP heritability, we used six primary categories (coding, UTR, promoter, DHSs, intronic and intergenic) that were proposed by Gusev et al. ${ }^{27}$. All six functional categories were jointly analysed.

Data availability. Summary statistics of our genome-wide analysis can be downloaded from the Bio-X website (http://analysis.bio-x.cn/gwas/).

\section{References}

1. Osayande, A. S. \& Mehulic, S. Diagnosis and initial management of dysmenorrhea. Am. Fam. Physician 89, 341-346 (2014).

2. Dawood, M. Y. Primary dysmenorrhea: advances in pathogenesis and management. Obstet. Gynecol. 108, 428-441 (2006).

3. Proctor, M. \& Farquhar, C. Diagnosis and management of dysmenorrhoea Br. Med. J. 332, 1134-1138 (2006).

4. Gagua, T., Tkeshelashvili, B., Gagua, D. \& McHedlishvili, N. Assessment of anxiety and depression in adolescents with primary dysmenorrhea: a case-control study. J. Pediatr. Adolesc. Gynecol. 26, 350-354 (2013).

5. Wang, L. et al. Stress and dysmenorrhoea: a population based prospective study. Occup. Environ. Med. 61, 1021-1026 (2004).

6. Chan, W. Y., Dawood, M. Y. \& Fuchs, F. Prostaglandins in primary dysmenorrhea. Comparison of prophylactic and nonprophylactic treatment with ibuprofen and use of oral contraceptives. Am. J. Med. 70, 535-541 (1981).

7. Coco, A. S. Primary dysmenorrhea. Am. Fam. Physician 60, 489-496 (1999).

8. Silberg, J. L., Martin, N. G. \& Heath, A. C. Genetic and environmental factors in primary dysmenorrhea and its relationship to anxiety, depression, and neuroticism. Behav. Genet. 17, 363-383 (1987).

9. Treloar, S. A., Martin, N. G. \& Heath, A. C. Longitudinal genetic analysis of menstrual flow, pain, and limitation in a sample of Australian twins. Behav. Genet. 28, 107-116 (1998).

10. Zhang, J. The Study of the Genetic Factor and Pesticide Exposure on Primary Dysmenorrhea. Master Thesis, Anhui Medical Univ. (2002).

11. Lee, L.-C. et al. Association of brain-derived neurotrophic factor gene Val66Met polymorphism with primary dysmenorrhea. PLoS ONE 9, e112766 (2014).

12. Ozsoy, A. Z. et al. The evaluation of IL6 and ESR1 gene polymorphisms in primary dysmenorrhea. Immunol. Invest. 45, 75-86 (2016).

13. Marconi, S. et al. Human adipose-derived mesenchymal stem cells systemically injected promote peripheral nerve regeneration in the mouse model of sciatic crush. Tissue Eng. A 18, 1264-1272 (2012).

14. Berg, J. et al. Human adipose-derived mesenchymal stem cells improve motor functions and are neuroprotective in the 6-hydroxydopamine-rat model for Parkinson's disease when cultured in monolayer cultures but suppress hippocampal neurogenesis and hippocampal memory function when cultured in spheroids. Stem Cell Rev. Rep. 11, 133-149 (2015).

15. Gonzalez-Rey, E. et al. Human adipose-derived mesenchymal stem cells reduce inflammatory and $\mathrm{T}$ cell responses and induce regulatory $\mathrm{T}$ cells in vitro in rheumatoid arthritis. Ann. Rheum. Dis. 69, 241-248 (2010).

16. Uccelli, A., Moretta, L. \& Pistoia, V. Mesenchymal stem cells in health and disease. Nat. Rev. Immunol. 8, 726-736 (2008). 
17. Garland, E. L. Pain processing in the human nervous system a selective review of nociceptive and biobehavioral pathways. Prim. Care 39, 561-571 (2012).

18. Li, X., Thyssen, G., Beliakoff, J. \& Sun, Z. The novel PIAS-like protein hZimp10 enhances Smad transcriptional activity. J. Biol. Chem. 281, 23748-23756 (2006).

19. Wan, Y. Y. \& Flavell, R. A. TGF-beta and regulatory T cell in immunity and autoimmunity. J. Clin. Immunol.J. Clin. Immunol. 28, 647-659 (2008).

20. Jones, A. V. et al. Genome-wide association analysis of pain severity in dysmenorrhea identifies association at chromosome 1p13.2, near the nerve growth factor locus. Pain 157, 2571-2581 (2016).

21. Aloe, L. Nerve growth factor and neuroimmune responses: basic and clinica observations. Arch. Physiol. Biochem. 109, 354-356 (2001).

22. Datta-Mitra, A., Kundu-Raychaudhuri, S., Mitra, A. \& Raychaudhuri, S. P. Cross talk between neuroregulatory molecule and monocyte: nerve growth factor activates the inflammasome. PLoS ONE 10, e0121626 (2015).

23. Raychaudhuri, S. P., Raychaudhuri, S. K., Atkuri, K. R., Herzenberg, L. A. \& Herzenberg, L. A. Nerve growth factor A key local regulator in the pathogenesis of inflammatory arthritis. Arthritis Rheum. 63, 3243-3252 (2011).

24. Raychaudhuri, S. P., Jiang, W.-Y. \& Raychaudhuri, S. K. Revisiting the Koebner phenomenon-role of NGF and its receptor system in the pathogenesis of psoriasis. Am. J. Pathol. 172, 961-971 (2008).

25. Bullo, M., Peeraully, M. R. \& Trayhurn, P. Stimulation of NGF expression and secretion in 3T3-L1 adipocytes by prostaglandins $\mathrm{PGD}_{2}, \mathrm{PGJ}_{2}$, and $\Delta^{12}-\mathrm{PGJ}_{2}$. Am. J. Physiol. Endocrinol. Metab. 289, E62-E67 (2005).

26. McMahon, S. B. NGF as a mediator of inflammatory pain. Philos. Tranasac. $R$. Soc. Lond. B Biol. Sci. 351, 431-440 (1996).

27. Gusev, A. et al. Partitioning heritability of regulatory and cell-type-specific variants across 11 common diseases. Am. J. Hum. Genet. 95, 535-552 (2014).

28. Sapkota, Y. et al. Association between endometriosis and the interleukin $1 \mathrm{~A}$ (ILIA) locus. Hum. Reprod. 30, 239-248 (2015).

29. Welter, D. et al. The NHGRI GWAS Catalog, a curated resource of SNP-trait associations. Nucleic Acids Res. 42, D1001-D1006 (2014).

30. Kuno, K. \& Matsushima, K. The IL-1 receptor signaling pathway. J. Leuk. Biol. 56, 542-547 (1994)

31. Martinez, V. G. et al. The canonical BMP signaling pathway is involved in human monocyte-derived dendritic cell maturation. Immunol. Cell Biol. 89, 610-618 (2011)

32. Sapkota, Y. et al. Independent replication and meta-analysis for endometriosis risk loci. Twin. Res. Hum. Genet. 18, 518-525 (2015).

33. Yang, J. et al. Genome partitioning of genetic variation for complex traits using common SNPs. Nat. Genet. 43, 519-525 (2011).

34. Lee, S. H. et al. Estimating the proportion of variation in susceptibility to schizophrenia captured by common SNPs. Nat. Genet. 44, 247-250 (2012)

35. Revill, S. I., Robinson, J. O., Rosen, M. \& Hogg, M. I. The reliability of a linear analogue for evaluating pain. Anaesthesia 31, 1191-1198 (1976).

36. Larroy, C. Comparing visual-analog and numeric scales for assessing menstrual pain. Behav. Med. 27, 179-181 (2002).

37. Delaneau, O., Marchini, J. \& Zagury, J.-F. A linear complexity phasing method for thousands of genomes. Nat. Methods 9, 179-181 (2012).

38. Howie, B. N., Donnelly, P. \& Marchini, J. A flexible and accurate genotype imputation method for the next generation of genome-wide association studies. PLos Genet. 5, e1000529 (2009).

39. Altshuler, D. M. et al. An integrated map of genetic variation from 1,092 human genomes. Nature 491, 56-65 (2012).

40. Price, A. L. et al. Principal components analysis corrects for stratification in genome-wide association studies. Nat. Genet. 38, 904-909 (2006).

41. Marchini, J., Howie, B., Myers, S., McVean, G. \& Donnelly, P. A new multipoint method for genome-wide association studies by imputation of genotypes. Nat. Genet. 39, 906-913 (2007).

42. Shi, Y. Y. \& He, L. SHEsis, a powerful software platform for analyses of linkage disequilibrium, haplotype construction, and genetic association at polymorphism loci. Cell Res. 15, 97-98 (2005)

43. Shen, J. et al. SHEsisPlus, a toolset for genetic studies on polyploid species. Sci. Rep. 6, 24095 (2016).

44. Liu, J. Z. et al. Meta-analysis and imputation refines the association of $15 \mathrm{q} 25$ with smoking quantity. Nat. Genet. 42, 436-440 (2010).

45. Ward, L. D. \& Kellis, M. HaploReg: a resource for exploring chromatin states, conservation, and regulatory motif alterations within sets of genetically linked variants. Nucleic Acids Res. 40, D930-D934 (2012).
46. Boyle, A. P. et al. Annotation of functional variation in personal genomes using RegulomeDB. Genome Res. 22, 1790-1797 (2012).

47. Dunham, I. et al. An integrated encyclopedia of DNA elements in the human genome. Nature 489, 57-74 (2012).

48. Kundaje, A. et al. Integrative analysis of 111 reference human epigenomes. Nature 518, 317-330 (2015).

49. Zhou, X. et al. Epigenomic annotation of genetic variants using the Roadmap Epigenome Browser. Nat. Biotechnol. 33, 345-346 (2015).

50. Hindorff, L. A. et al. Potential etiologic and functional implications of genomewide association loci for human diseases and traits. Proc. Natl Acad. Sci. USA 106, 9362-9367 (2009).

51. Mishra, A. \& Macgregor, S. VEGAS2: software for more flexible gene-based testing. Twin. Res. Hum. Genet. 18, 86-91 (2015)

52. Segre, A. V. et al. Common inherited variation in mitochondrial genes is not enriched for associations with type 2 diabetes or related glycemic traits. PLoS Genet. 6, e1001058 (2010).

53. Perry, J. R. B. et al. Parent-of-origin-specific allelic associations among 106 genomic loci for age at menarche. Nature 514, 92-97 (2014).

54. Yang, J., Lee, S. H., Goddard, M. E. \& Visscher, P. M. GCTA: a tool for genome-wide complex trait analysis. Am. J. Hum. Genet. 88, 76-82 (2011).

\section{Acknowledgements}

We are deeply grateful to all the participants who contributed to this project. This work was supported by the National Key Basic Research Program of China (973 Program; 2015CB559100), the National Key R\&D Program - Special Project on Precision Medicine (2016YFC0903402), the National Natural Science Foundation of China (31325014, 81421061), the Program of Shanghai Academic Research Leader (15XD1502200), National Program for Support of Top-Notch Young Professionals, Shanghai Key Laboratory of Psychotic Disorders (13dz2260500), the China Postdoctoral Science Foundation (2016M590615, 2016M600334), the Shandong Postdoctoral Innovation Foundation (201601015), the Qingdao Postdoctoral Application Research Project (2016048).

\section{Author contributions}

Y.S. conceived, designed and supervised the study, and obtained financial support; Y.Z Y.W., J.X., J.J., J.S., W.Z., Z.C., Q.S., L.M., B.Y., D.Z., Y.X., Y.Z., D.L., Y.S. and W.Z. participated in sample collection and phenotyping; J.C., J.J., J.S., Y.X., Y.Z., D.L., Y.S., W.Z. and C.L. performed sample processing and involved in data management; Z.L. and J.S. conducted bioinformatics/statistical analyses; Y.S. and Z.L. interpreted the data and drafted the manuscript. All authors critically reviewed the article and approved the final manuscript.

\section{Additional information}

Supplementary Information accompanies this paper at http://www.nature.com/ naturecommunications

Competing interests: The authors declare no competing financial interests.

Reprints and permission information is available online at http://npg.nature.com/ reprintsandpermissions/

How to cite this article: $\mathrm{Li}, \mathrm{Z}$. et al. Common variants in ZMIZ1 and near NGF confer risk for primary dysmenorrhoea. Nat. Commun. 8, 14900 doi: 10.1038/ncomms14900 (2017).

Publisher's note: Springer Nature remains neutral with regard to jurisdictional claims in published maps and institutional affiliations.

This work is licensed under a Creative Commons Attribution 4.0 International License. The images or other third party material in this article are included in the article's Creative Commons license, unless indicated otherwise in the credit line; if the material is not included under the Creative Commons license, users will need to obtain permission from the license holder to reproduce the material To view a copy of this license, visit http://creativecommons.org/licenses/by/4.0/

(C) The Author(s) 2017 